\title{
UMA EVIDÊNCIA DA DISCRIMINAÇÃO SALARIAL NO ESTADO DO RIO GRANDE DO SUL
}

\section{Kalinca Leia Becker ${ }^{1}$ \\ Darcy Ramos da Silva Neto \\ Fernando Cavalheiro Krauzer ${ }^{3}$}

Resumo: $\bigcirc$ objetivo principal desse estudo é analisar as diferenças salariais de gênero e etnia no Estado do Rio Grande do Sul. Para isso, foram estimadas equações de rendimentos com base nas informações da PNAD (2015). Os resultados indicaram que, em média, os homens recebem um salário/hora 18,41\% maior em relação às mulheres. Já as pessoas autodeclaradas da cor branca recebem, em média, $9,58 \%$ a mais por hora de trabalho. A vantagem salarial desses grupos também é observada quando são considerados individuos com o mesmo nivel educacional.

Palavras-chave: Gênero; etnia; discriminação salarial.

\begin{abstract}
This article is a proposal to bring up the relevant questions concerning income differentiation beyond individual qualifications of productive aspect, such as ethnicity and gender. The analysis is made for the year 2015, with the attempt to explain a parameter of the gender and ethnicity wage differences in the State of Rio Grande do Sul using data from the PNAD. The main results of the analysis converge with the theoretical reference: there is still wage discrimination, especially to factors related to ethnicity and gender, also measured by the same educational level.
\end{abstract}

Keywords: Gender; ethnicity; salary discrimination.

\section{Um Breve Panorama sobre Discriminação Salarial no Brasil}

\section{1 - Discriminação por gênero}

Diversos estudos relatam que a forma de inserção no mercado de trabalho para homens e mulheres é dada de maneiras distintas e, no Brasil, essa relação é significativamente acentuada.

Quando se trata de qualificações, ocupações semelhantes e também

\footnotetext{
1 Doutora em Economia Aplicada ESALQ-USP e Professora Adjunta no Departamento de Economia e Relações Internacionais da Universidade Federal de Santa Maria. E-mail: kalincabecker@gmail.com.

2 Mestrando em Economia na Universidade Federal de Santa Maria e pesquisador assistente no Instituto de Pesquisa Econômica Aplicada (IPEA). E-mail: darcy.neto@ipea.gov.br.

3 Mestrando em Economia e Desenvolvimento na Universidade Federal de Santa Maria. E-mail: fe_krauzer@ hotmail.com.
} 
indivíduos que moram na mesma região, homens são mais bem remunerados por hora e tendem a cumprir jornadas maiores de trabalho, o que acentua ainda mais a diferença salarial. Além disso, há também um elevado grau de segregação ocupacional, ou seja, a maioria dos cargos é preenchido por homens ou mulheres, e não por ocupações mistas, como apontam Cavalieri e Fernandes (1998) e Barros, Ramos e Santos (1992) apud Barros et al. (2001).

Após a década de 1970, a participação das mulheres no mercado de trabalho aumentou significativamente. Em 1999, as mulheres representavam cerca de 41\% da população economicamente ativa e recebiam, em média, pouco mais da metade do salário dos homens, aproximadamente $60,7 \%$. A diferença dos rendimentos entre homens e mulheres pode ser explicada com base nas características médias destes dois grupos, como por exemplo: escolaridade, experiência, idade e ramo de trabalho. Porém, tais características explicam apenas parte da diferença salarial, sendo o restante atribuído a um componente residual associado à discriminação (GIUBERTI \& FILHO, 2005).

Para Cavalieri e Fernandes (1998), os salários dos homens eram, em média, $58,38 \%$ maiores que os das mulheres em 1989, e, além disso, quando são adicionados alguns controles para tentar explicar essa diferença, ele permanece constante devido ao componente residual já mencionado por Giuberti e Filho (2005).

Em 1990, década que foi caracterizada pela intensa abertura comercial, baixos niveis de investimento e ampla terceirização da economia, houve uma contínua tendência da incorporação da mulher no mercado de trabalho. Incrementa-se, nesta mesma década, o desemprego feminino, o que indica que o aumento dos postos de trabalho não foi suficiente para absorver a totalidade do crescimento da população feminina economicamente ativa (HOFFMANN \& LEONE, 2004).

Para Barros et al. (2001), nos casos dos diferenciais homem-mulher na questão de empregabilidade no Brasil, observa-se que há pouca distinção de experiência. Sendo que mulheres são sensivelmente mais escolarizadas e homens têm tido melhores condições de trabalho. Essa diferenciação pode ser vista por três aspectos: (i) custo de oportunidade do tempo utilizado é diferente entre homens e mulheres com atividades não-laborais (cuidar da casa, filhos, preferências socioculturais ou biológicas); (ii) uma discriminação pura e simples entre gêneros e oportunidade de cargos (observa-se que os "insumos", nesse caso, são aproximadamente iguais, ou seja, uma força de trabalho, em média, igual); e, (iii) a mão-de-obra entre homens e mulheres pode ser vista pelas firmas como diferentes fatores de produção, ou seja, a produtividade e quantidade demandada de homens e mulheres com idênticos estoques de capital humano, podem ser analisados de maneiras diferentes de acordo com o grau de complementaridade com os demais fatores de produção.

Adentrando nas questões de cônjuge e filhos, definido acima como "atividades 
não-laborais", Wajnman (2016) aponta que há diferenciação das chances da atuação da mulher e do homem no mercado de trabalho. Para o homem, ter cônjuge e/ou filhos (tanto em idade pré-escolar quanto em idade escolar) aumenta suas chances de atuação econômica no mercado de trabalho, a razão de chance de participação econômica do homem que tem cônjuge é de 1,85; filho em idade pré-escolar é de 1,25; e 1,15 em idade escolar. Destarte, para a mulher a situação é inversa: sua participação econômica reduz quando existe a figura de cônjuge e/ou filhos: a razão da chance da mulher no mercado de trabalho é 0,44 quando tem cônjuge; 0,60 quando tem filho em idade pré-escolar e 0,80 quando tem filho em idade escolar. Além dos estudos que foram feitos pela autora com outras categorias: a razão da mulher para atuação econômica é maior que a do homem em trabalhos precários, parciais e autônomos, para as mesmas três situações apresentadas (filhos em idade pré-escolar, idade escolar e cônjuge). Por fim, é apresentado um modelo de Heckman para o log do salário hora por sexo para a realidade brasileira de acordo com a PNAD 2014. Dado as mesmas três situações apresentadas, as mulheres possuem em média salários-hora menores que os homens, o que a literatura denomina como prêmio do casamento e da paternidade no mercado de trabalho.

Sachsida e Loureiro (1998) apud Barros et al. (2001) estimam que existe uma elasticidade-preço cruzada negativa da demanda por trabalho feminino, ou seja, um aumento do salário médio dos homens causa uma redução no nível de emprego das mulheres. De fato, o fator de produção de capacidade humana deveria ser igual entre homens e mulheres, trazendo para o conceito microeconômico, eles deveriam ser "substitutos perfeitos" para as empresas, porém, na presença de discriminação no mercado de trabalho, homens e mulheres não são vistos como forças de trabalho iguais.

É necessário também levar em consideração o segmento de mercado em que o indivíduo está inserido. Silva e Kassouf (2000) apontam que a discriminação é significativa em ambos os segmentos, formais e informais, porém, é ainda maior no setor formal do mercado de trabalho. Conforme os autores, se não houvesse discriminação no mercado formal, os rendimentos das mulheres aumentariam em aproximadamente $47 \%$, o que atingiria valores superiores aos dos homens em $15 \%$. Os dados apresentados no mercado informal são ainda mais preocupantes, pois as características individuais explicam 3\% das diferenças dos rendimentos, enquanto os outros $97 \%$ são explicados pela discriminação.

No que diz respeito às ocupações menos valorizadas e, tradicionalmente femininas no mercado de trabalho, há uma continuidade de nichos ocupacionais, como por exemplo, o trabalho doméstico. Essa condição reflete um aumento da proporção de mulheres no mercado informal, desprotegidas de qualquer regulamentação que lhes garantam direitos sociais e trabalhistas. Portanto, vale ressaltar que o aumento das ocupações precárias também tem ajudado à 
absorção da parcela dos homens, causando uma redução da segmentação por gênero. Assim, as diferenças de trabalho masculino e feminino têm diminuído não somente pela capacidade das mulheres de entrarem no mercado de trabalho que era reservado aos homens, mas também pela participação de homens e mulheres nos empregos precários que o mercado costuma oferecer a ambos os sexos (LEONE, 2003).

Para Hoffmann e Leone (2004), existe uma forte dificuldade das mulheres encontrarem alternativas de emprego que possam desloca-las para outro setor que fosse melhor remunerado. A permanência das mulheres nesses primeiros empregos transitórios dificulta a inserção das mulheres economicamente ativas no mercado de trabalho. Existem alguns setores com niveis ocupacionais mais elevados que as mulheres estão inseridas, em sua maioria, como as atividades sociais (assistência social, educação, saúde e previdência) que podem ser vistos como serviços auxiliares das atividades econômicas de administração pública.

Contrapondo o que foi apresentado até aqui, para Lavinas (2001), é inegável - aumento da participação feminina no mercado brasileiro nos últimos 20 anos, porém, a participação feminina para a autora é tida de forma linear e alheia às flutuações das atividades econômicas. Dada uma situação de recessão ou expansão econômica, a taxa de participação das mulheres no mercado, em particular de cônjuges com filhos, vêm aumentando no país.

Há muitas razões para essa expansão da empregabilidade feminina em relação aos homens, decorrendo, de acordo com Lavinas (2001):

Uma delas decorre da amplitude do processo de reestruturação positiva iniciado na década de 90 e que afeta sobremaneira o emprego industrial, cuja redução "massiva" tem rebatimentos negativos, incidindo mais sobre os homens do que sobre as mulheres, já pouco representadas no setor. Outro fator a estimular a inserção produtiva das mulheres diz respeito à expansão da economia de serviços. verdade, entretanto, que esse fenômeno pouco tem alterado o grau de "mixidade" intra-setorial, dado o perfil da segregação ocupacional por gênero: no setor de serviços, as mulheres permanecem majoritárias (mais de 70\%) nas atividades de saúde e ensino (setor público e privado), na administração pública e nos serviços pessoais, atividades moldadas pelo tradicional lugar do feminino na esfera da reprodução. (LAVINAS, 2001).

Há uma terceira dinâmica que fomenta o aumento do emprego feminino, com uma maior flexibilização do mercado de trabalho e a precarização das relações de trabalho já mencionadas aqui. Isso explica o aumento das oportunidades de emprego das mulheres. Sobretudo, há algumas características herdadas da atual divisão de trabalho por sexo, como por exemplo, o fato de mulheres se submetem 
a empregos em tempo parcial para compatibilizar com o trabalho doméstico, trabalhos secundários que gerariam rendimentos menores que dos homens que, em face do aumento do desemprego, seriam as primeiras a serem dispensadas, devido ao baixo poder de barganha. Na verdade, há um trade-off entre a elevação da taxa de emprego feminino e a precarização das relações de trabalho, o que pode ser uma explicação para as vantagens comparativas da mão-de-obra feminina em relação à masculina (LAVINAS, 2001).

Outro contraponto importante é a análise de que a participação feminina na força de trabalho amplia o efeito de seus rendimentos sobre a desigualdade de renda domiciliar. Portanto, tal efeito depende da correlação entre os rendimentos femininos e os demais. Podem ser evidenciadas por Hoffmann e Leone (2004) duas situações extremas: (i) caso o aumento da participação feminina ocorrer apenas com mulheres com altos rendimentos de domicilios de alta renda, então a desigualdade domiciliar irá aumentar com a participação feminina; (ii) caso contrário, se o aumento da participação feminina deve-se a mulheres de domicilios de baixa renda que irão auferir rendimentos baixos, então irá ocorrer uma redução da desigualdade da renda domiciliar com a maior participação da mulher.

Com este estudo de caso feito por Hoffmann e Leone (2004), os autores chegaram à conclusão de que, nas últimas décadas, a participação da mulher nas atividades econômicas continuou aumentando. $A$ intensificação da participação das mulheres elevou-se a proporção de domicilios com mulheres na força de trabalho. Ou seja, uma maior participação feminina e maior contribuição dos rendimentos para a renda domiciliar. Para concluir, deve-se salientar também as recentes alterações na composição do rendimento familiar, diminuindo a parcela de contribuição do homem e aumentando a participação da mulher.

\section{2 - Discriminação por Etnia}

Para Loureiro (2003), a discriminação no mercado de trabalho pode ser classificada por quatro tipos: discriminação salarial, discriminação de emprego, discriminação ocupacional e discriminação ao acesso do capital humano. Sendo que a discriminação salarial significa que trabalhadores do sexo feminino (e negros) recebem salários menores do que homens (brancos) que possuem o mesmo trabalho. Discriminação de emprego ocorre quando mulheres e negros ficam em completa desvantagem em relação à baixa oferta de emprego, portanto, tendo um maior índice de desemprego. Discriminação ocupacional significa dizer que mulheres (e negros) têm sido arbitrariamente restringidos de ocupar determinados cargos, mesmo sendo tão capaz quanto qualquer outro. E, discriminação decorrente de oportunidades desiguais para obtenção de capital humano, quando mulheres (e negros) possuem menores oportunidades de se capacitar e aumentar sua produtividade, como educação formal e/ou treinamento. 
Para Loureiro (2003), os três primeiros tipos são denominados como "discriminação direta", pois referem-se a indivíduos que já estão empregados. O último tipo é designado como "discriminação indireta", pois ocorre antes de o indivíduo se inserir no mercado de trabalho.

De acordo com o Instituto de Pesquisa Econômica Aplicada (IPEA, 2015), houve um aumento populacional nos últimos anos, e um aumento relevante da população negra (preta e parda) no país. Em 1995, 44,9\% dos brasileiros declaravam-se negros e, em 2009, este percentual subiu para $51,1 \%$. Enquanto a população branca (brancos e amarelos) caiu de $54,5 \%$ para $48,2 \%$ no mesmo periodo. Em 2009 , o percentual de homens brancos era de $47 \%$ e $52 \%$ o percentual de homens negros, mulheres brancas era de $49,3 \%$ e mulheres negras $49,9 \%$. Porém, tais alterações estão, em parte, associadas a uma mudança na estrutura de auto declaração das pessoas como pertencentes a tais grupos de cor/etnia.

Apesar da maior inserção da população negra no mercado de trabalho nas últimas décadas, um estudo feito pelo IPEA (2015) mostrou que a taxa de desemprego da população com 16 anos de idade ou mais era de 5,3\% para homem branco, 6,6\% para homem negro, para 9,2\% mulher branca, e 12,5\% para mulher negra.

De acordo com um estudo realizado por Campante et al. (2004), o mercado de trabalho para uma pessoa negra é mais favorável no serviço público, sendo a pior situação a de um trabalhador sem carteira assinada. Dado que exista um negro capacitado pelas vias legais de atender a um cargo público, não há razões para discriminação. Portanto, é muito mais vantajoso para uma pessoa negra entrar no serviço público, mas a proporção de negros no serviço público é significativamente menor que a parcela de brancos, esbarrando na questão da desigualdade do acesso à educação, entre outros fatores, que Sen (2001) trata como privações das capacitações dos indivíduos.

Para Henriques (2001), a escolaridade entre brancos e negros expõe a inércia do padrão de discriminação racial no país. Apesar da melhoria dos níveis médios de escolaridade ao longo do século, o padrão de discriminação se mantém praticamente estável entre as gerações. Afinal, como destaca Soares (2000), a concretização do salário do indivíduo está dividida em três etapas: formação, inserção e definição salarial. Para Campante et al. (2004), em todas as três etapas os negros são prejudicados, tanto no acesso à educação, inserção no mercado de trabalho e o poder de barganha de salários. Isso se deve a discriminação e também ao fato de que, em média, os negros são menos escolarizados.

O Brasil é um país bastante heterogêneo do ponto de vista regional, ou seja, não é esperado que o grau de discriminação do mercado de trabalho seja o mesmo entre todas as regiões do país. $O$ conhecimento mais detalhado do perfil regional é, sem dúvida, relevante, seja com o intuito de obter um retrato mais preciso ou até mesmo o desenho de políticas públicas mais adequadas (CAMPANTE et 
al., 2004). Exatamente com base neste ponto, o Estado do Rio Grande do Sul apresenta-se como um estudo interessante e relevante, afinal, historicamente, - Estado contou com a utilização do trabalho escravo de origem africana nas fazendas de charque. Posteriormente, recebeu muitos imigrantes de origem europeia, o que pode estar associado a grande concentração de população branca no Estado. Tais especificidades na composição da população podem fazer com que a discriminação se manifeste de forma diferenciada da média nacional.

\section{Metodologia}

Esta seção é dividida em duas subseções. A primeira parte detalha o quesito metodológico do trabalho empírico, contendo a descrição das variáveis de interesse e controles, a variável dependente e os modelos a serem estimados. A outra parte apresenta uma análise descritiva dos dados e reporta a significância estatística das variáveis e os sinais obtidos dos coeficientes.

\section{Aspectos Metodológicos}

A análise que predomina nos determinantes salariais com foco nos componentes de gênero e etnia envolve a estimação de modelo do tipo crosssection a partir do Método dos Mínimos Quadrados Ordinários (MQO). Conforme destaca Wooldridge (2010), os dados de corte transversal consistem em uma amostra de indivíduos, empresas, cidades, países ou uma multiplicidade de outras unidades, tomada para um determinado ponto no tempo. $O$ emprego desta forma de dados é importante para testar e avaliar políticas governamentais.

Para este artigo, foi selecionada a Pesquisa Nacional por Amostra de Domicílios - PNAD (IBGE, 2015) para o Estado do Rio Grande do Sul. Conforme mencionado anteriormente, este trabalho visa evidenciar empiricamente a relação salarial com o gênero e etnia das pessoas do Estado.

De acordo com as tabelas 1 e 2 , verifica-se que existe uma variável resposta, Tabela 1 - Nomenclaturas, Definições e Fonte de dados - Variável Resposta

\begin{tabular}{|c|l|r|}
\hline Nomenclaturas & \multicolumn{1}{|c|}{ Definição } & Fonte de dados \\
\hline \multirow{3}{*}{ RENDAH } & $\begin{array}{l}\text { Relação da renda que o individuo } \\
\text { ganha mensalmente com a } \\
\text { quantidade de horas que o mesmo } \\
\text { trabalha durante o mês. Esta variável } \\
\text { será analisada na relação renda/hora. }\end{array}$ & PNAD 2015 \\
\hline
\end{tabular}

Fonte: Elaborado pelos autores em 2017 a partir dos dados da PNAD 2015. 
Tabela 2 - Nomenclaturas, Definições e Fonte de dados - Variáveis Independentes

\begin{tabular}{|c|c|c|}
\hline Nomenclaturas & Definição & Fonte de dados \\
\hline GEN & $\begin{array}{l}\text { A variável possui uma combinação binária entre } \\
\text { gêneros. Se for homem, é atribuído o valor } 1 \text {, se } \\
\text { for mulher, é atribuído o valor } 0 \text {. }\end{array}$ & \multirow{10}{*}{ PNAD 2015} \\
\hline $\mathrm{ETN}^{4}$ & $\begin{array}{l}\text { A variável possui uma combinação binária entre } \\
\text { etnia. Como a PNAD utiliza auto-declaração de } 5 \\
\text { categorias, para efeito de simplificação, foi usado } \\
\text { o valor } 1 \text { para branco (branco e amarelo) e } 0 \text { para } \\
\text { não-branco (preto, pardo, indígena). }\end{array}$ & \\
\hline EDUC & $\begin{array}{l}\text { Nível de escolaridade do indivíduo medida em } \\
\text { anos. }\end{array}$ & \\
\hline $\begin{array}{l}\text { PERM, } \\
\text { ou variável dep }\end{array}$ & $\begin{array}{l}\text { Permanência, medida em anos, do indivíduo em } \\
\text { ndentráneis independentes, ou re }\end{array}$ & \\
\hline EXPER & $\begin{array}{l}\text { Experiência, medida em anos, do indivíduo ao } \\
\text { longo de sua carreira profissional. }\end{array}$ & \\
\hline FILH & Número de filhos. & \\
\hline $\mathrm{SET}^{5}$ & $\begin{array}{l}\text { Setor do emprego atual do indivíduo, uma } \\
\text { combinação binária que atribui valor } 1 \text { para setor } \\
\text { público e } 0 \text { para setor privado. }\end{array}$ & \\
\hline $\mathrm{CDM}^{6}$ & $\begin{array}{l}\text { Condição de Moradia do indivíduo. Uma } \\
\text { atribuição binária sendo } 1 \text { se mora em área urbana } \\
\text { e } 0 \text { se mora em área rural. }\end{array}$ & \\
\hline CUD & $\begin{array}{l}\text { Condição na unidade domiciliar. Uma condição } \\
\text { binária sendo o valor } 1 \text { atribuído para o chefe da } \\
\text { família e } 0 \text { caso contrário. }\end{array}$ & \\
\hline POT & $\begin{array}{l}\text { Posição na ocupação do trabalho. Uma atribuição } \\
\text { binária sendo } 1 \text { o indivíduo que é empregador e } 0 \\
\text { caso contrário. }\end{array}$ & \\
\hline
\end{tabular}

Fonte: Elaborado pelos autores em 2017 a partir dos dados da PNAD 2015.

\footnotetext{
$4 \quad$ Há um ponto na definição das categorias de etnia que precisam ser explicado: A PNAD usa a auto-declaração, que são agrupados as seguintes categorias: brancos, negros, pardos, amarelos e indígenas. Para o presente estudo, a definição de etnia será feita em duas categorias: brancos (branc

5 Ainda existem outros setores de trabalho, a PNAD (2015) contabiliza também trabalho autônomo, trabalho informal, entre outros segmentos e formatos. A análise de apenas setor público e setor privado foram escolhidos para simplificação do modelo, pois a particularidade do artigo está em evidenciar diferenças salariais principalmente entre gênero e etnia.

6 Existem 8 subdivisões para áreas urbanas e rurais segundo a PNAD (2015), 3 para áreas urbanas e 5 para áreas rurais, mas, também para simplificação do modelo, está sendo considerado apenas área urbana e área rural.
} 
Para a análise das variáveis que possivelmente afetam a renda, entre fatores produtivos e não produtivos, serão propostos 5 modelos gerais neste trabalho, sendo que o modelo seguinte sempre incorpora uma variável adicional em relação ao modelo anterior. Essa estrutura de análise segue a proposta de Alves e Vieira (2011), que analisam o comportamento dos parâmetros estimados das variáveis de interesse na medida em que o modelo vai ficando mais complexo.

Para o primeiro modelo de regressão por $\mathrm{MQO}$, temos:

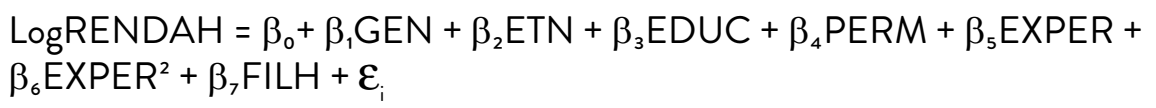

para

$\mathrm{i}=1,2 . . \mathrm{N} ; \mathrm{N}$ sendo o números de indivíduos da amostra.

Note que foi especificado, já de início, um modelo no formato logaritmolinear, ou "log-lin", pois o intuito proposto é mensurar a sensibilidade das variáveis em relação ao salário/hora. $O$ percentual que o indivíduo ganha a mais ou a menos, em média e ceteris paribus, dada a variável independente a ser analisada.

Cabe salientar também que o modelo está incorporando o termo quadrático da experiência profissional do indivíduo $\left(E X P E R^{2}\right.$ ), para que se possa captar - efeito parcial desta variável no salário/hora, em outras palavras, até em que momento da experiência do indivíduo o salário continua aumentando marginalmente. Desse modo, buscando identificar quantos anos são necessários para que o indivíduo atinja o auge salarial devido sua experiência profissional e a partir de que momento, em média, seus aumentos salariais marginais começam a cair (ponto de viragem, ou turnning point).

Conforme já mencionado, a variável dependente y (LogRENDAH) é o ganho monetário do individuo por hora (salário/hora), $\beta_{0}$ é a constante, $\beta_{1}$ e $\beta_{2}$ são os parâmetros das variáveis dummy que captam a relação dos homens e mulheres com o salário/hora e a relação da etnia entre brancos e não brancos com o salário/hora, respectivamente e medidos em percentual. $\beta_{4}$ e $\beta_{5}$ são, respectivamente, os parâmetros relacionados aos anos que o indivíduo está no emprego atual e os anos de sua experiência profissional. Espera-se que ambos apresentem relações positivas com a variável resposta.

$\beta_{6}$ é o termo quadrático do modelo para explicar o ponto crítico da relação 
entre experiência profissional e salário/hora. Quando o coeficiente de $x_{i}$ é positivo e o coeficiente de $x_{i}^{2}$ é negativo, a função quadrática tem um formato parabólico e sempre tem um valor positivo de $x_{i}$, no qual o efeito de $x_{i}$ sobre $y_{i}$ é zero; antes desse ponto, $x_{i}$ tem um efeito positivo sobre $y_{i}$; após esse ponto, $x_{i}$ tem um efeito negativo sobre $y_{i}$ (WOOLDRIDGE, 2010). $\beta_{7}$ é descrito, em unidades, como o parâmetro relacionado ao número de filhos que cada indivíduo possui e, por fim, $\varepsilon_{i}$ é o termo de erro da regressão.

modelo 2 assemelha-se ao primeiro modelo, porém, conforme já mencionado, conta com a adição de mais uma variável explicativa:

$\operatorname{LogRENDAH}=\beta_{0}+\beta_{1} \mathrm{GEN}+\beta_{2}$ ETN $+\beta_{3}$ EDUC $+\beta_{4}$ PERM $+\beta_{5}$ EXPER + $\beta_{6}$ EXPER $^{2}+\beta_{7} \mathrm{FILH}+\beta_{8} \mathrm{SET}+\eta_{\mathrm{i}}$

(2)

onde $\beta_{8}$ é o parâmetro e SET é a variável do setor em que o indivíduo trabalha, que também foi determinado por uma relação binária de 1 para o setor público e zero para o setor privado.

Seguindo a mesma lógica, o modelo 3 é estimado também pela adição de mais uma variável independente do modelo em relação ao modelo anterior:

$\operatorname{LogRENDAH}=\beta_{0}+\beta_{1} \mathrm{GEN}+\beta_{2}$ ETN $+\beta_{3}$ EDUC $+\beta_{4}$ PERM $+\beta_{5}$ EXPER + $\beta_{6}$ EXPER $2+\beta_{7}$ FILH $+\beta_{8}$ SET $+\beta_{9}$ CDM $+\omega_{i}$

onde $\beta_{9}$ é o parâmetro e a variável explicativa é a condição de moradia do indivíduo. $O$ intuito é detectar o indivíduo que mora em zona urbana em relação à renda e o indivíduo que mora na zona rural em relação à mesma.

modelo 4 pode ser descrito como:

$\operatorname{LogRENDAH}=\beta_{0}+\beta_{1}$ GEN $+\beta_{2}$ ETN $+\beta_{3}$ EDUC $+\beta_{4}$ PERM $+\beta_{5}$ EXPER + $\beta_{6}$ EXPER $^{2}+\beta_{7}$ FILH $+\beta_{8}$ SET $+\beta_{9}$ CDM $+\beta_{10}$ CUD $+\omega_{i}$

onde a variável CUD é a condição na unidade domiciliar do indivíduo e identifica o indivíduo que é o chefe de família.

Por fim, o modelo 5 irá englobar, a partir do modelo 4, mais uma variável 
explicativa. Portanto, sendo estimado como:

LogRENDAH $=\beta_{0}+\beta_{1}$ GEN $+\beta_{2}$ ETN $+\beta_{3}$ EDUC $+\beta_{4}$ PERM $+\beta_{5}$ EXPER + $\beta_{6}$ EXPER $^{2}+\beta_{7}$ FILH $+\beta_{8}$ SET $+\beta_{9}$ CDM $+\beta_{10}$ CUD $+\beta_{11}$ POT $+\vartheta i$

(5)

onde $\beta_{11}$ é o parâmetro associado a variável POT, que identifica o indivíduo que é empregador.

Após a análise dos resultados dos modelos de 1 a 5 , será analisado um modelo adicional, separadamente dos modelos gerais, a fim de contemplar alguma especificidade na relação salarial entre etnia e gênero. Segue o modelo adicional:

$\operatorname{LogRENDAH}=\varphi_{0}+\varphi_{1} \mathrm{GEN}{ }^{*} \mathrm{EDUC}+\varphi_{2} \mathrm{ETN}^{*} \mathrm{EDUC}+\tau_{\mathrm{i}}$ (6)

O modelo 6 busca compreender a diferença salarial (por hora) entre homens e mulheres e a diferença salarial (por hora) entre brancos e não brancos dado um ano a mais no nível de educação de ambos, onde $\varphi_{1}$ e $\varphi_{2}$ são os parâmetros e as novas variáveis que compreendem na multiplicação variável gênero pela variável educação subtraído da sua média. Da mesma forma a variável etnia, multiplicando-a pela educação menos a sua média. Segue a forma funcional da construção da variável:

GEN*EDUC $=$ GEN* $(E D U C-M E ́ D I A)$

(variável 11)

$E T N^{*} E D U C=E T N^{*}(E D U C-M E ́ D I A)$

(variável

12)

\section{Resultados}

A amostra da PNAD 2015 para o Estado do Rio Grande do Sul contempla 26.259 indivíduos que estão trabalhando e recebem salário. A Tabela 4 apresenta os resultados das estimações dos 5 modelos gerais especificados e suas análises parciais, os percentuais que cada variável, ceteris paribus, possui em relação com a renda/hora. A tabela 5 apresenta os resultados da estimação do modelo 6 . 
Tabela 4: Estimação dos Modelos 1 a 5

\begin{tabular}{|c|c|c|c|c|c|}
\hline Modelos & Modelo 1 & Modelo 2 & Modelo 3 & Modelo 4 & Modelo 5 \\
\hline \multirow{2}{*}{ CONSTANTE } & 2,1235 & 2,156 & 1,863 & 1,874 & 1,926 \\
\hline & $(69,23)^{\bullet \cdots \bullet}$ & $(70,75)^{\bullet \cdots}$ & $(50,96)^{\bullet \cdots *}$ & $(51,36)^{\bullet \cdots \bullet}$ & $(52,75)^{\bullet \cdots \bullet}$ \\
\hline \multirow{2}{*}{ GEN } & 0,1772 & 0,19377 & 0,203 & 0,180 & 0,169 \\
\hline & $(10,88)^{* * *}$ & $(11,98)^{* * \bullet}$ & $(12,68)^{* * *}$ & $(11,07)^{* * *}$ & $(10,46)^{* * *}$ \\
\hline \multirow{2}{*}{ ETN } & 0,0764 & 0,0835 & 0,094 & 0,0967 & 0,0915 \\
\hline & $(4,522)^{* * *}$ & $(4,982)^{* * *}$ & $(5,68)^{\iota \bullet \bullet}$ & $(5,822)^{4 * *}$ & $(5,54)^{* * *}$ \\
\hline \multirow{2}{*}{ EDUC } & 0,0983 & 0,091 & 0,0865 & 0,0862 & 0,0833 \\
\hline & $(53,36)^{* \cdots *}$ & $(48,16)^{* \ldots *}$ & $(45,57)^{*+*}$ & $(45,51)^{*+\cdots}$ & $(43,92)^{* * *}$ \\
\hline \multirow{2}{*}{ PERM } & 0,0138 & 0,0122 & 0,0141 & 0,0139 & 0,0131 \\
\hline & $(16,80)^{\star \cdots \bullet}$ & $(14,91)^{\bullet \cdots \bullet}$ & $(17,11)^{\bullet \cdots}$ & $(16,93)^{\bullet \cdots \bullet}$ & $(15,96)^{* \cdots *}$ \\
\hline \multirow{2}{*}{ EXPER } & 0,0266 & 0,0261 & 0,0255 & 0,0226 & 0,0218 \\
\hline & $(17,05)^{* * \bullet}$ & $(16,89)^{* * *}$ & $(16,63)^{* * *}$ & $(14,36)^{* * *}$ & $(13,91)^{* * *}$ \\
\hline \multirow[t]{2}{*}{ EXPER $^{2}$} & $-0,000388$ & $-0,000378$ & $-0,000369$ & $-0,000339$ & $=0,000330$ \\
\hline & $(-13,68)^{* * *}$ & $(-13,47)^{* 4 *}$ & $(-13,22)^{* 6 *}$ & $(-12,08)^{* 4 *}$ & $(-11,83)^{4 * 4}$ \\
\hline \multirow{2}{*}{ FILH } & $-0,0263$ & $-0,0255$ & $-0,0251$ & $-0,0244$ & $-0,261$ \\
\hline & $(-2,571)^{* *}$ & $(-2,516)^{* *}$ & $(-2,50)^{* \bullet}$ & $(-2,435)^{* *}$ & $(-2,618)^{* * *}$ \\
\hline \multirow{2}{*}{ SET } & & 0,317 & 0,313 & 0,3133 & 0,344 \\
\hline & & $(15,02)^{* * *}$ & $(14,94)^{* * *}$ & $(14,99)^{* * *}$ & $(16,45)^{* * *}$ \\
\hline \multirow{2}{*}{ CDM } & & & 0,342 & 0,338 & 0,325 \\
\hline & & & $(14,26)^{* * *}$ & $(14,12)^{* * *}$ & $(13,65)^{* * *}$ \\
\hline \multirow{2}{*}{ CUD } & & & & 0,112 & 0,1096 \\
\hline & & & & $(7,802)^{* * \bullet}$ & $(7,660)^{* \cdots}$ \\
\hline \multirow{2}{*}{ POT } & & & & & 0,424 \\
\hline & & & & & $(12,52)^{\bullet * \bullet}$ \\
\hline $\mathbf{R}^{2}$ & 0,255 & 0,268 & 0,280 & 0,284 & 0,293 \\
\hline Estatistica F & 613,07 & 574,29 & 541,33 & 495,62 & 470,44 \\
\hline
\end{tabular}

t-estatístico entre parênteses.

** $e^{* * *}$ indicam a significância, respectivamente, a $5 \%(1,96)$ e a $1 \%(2,58)$.

Fonte: Elaborado pelos autores em 2017 a partir dos dados da PNAD 2015.

Tabela 5: Estimação do Modelo $6^{7}$

\begin{tabular}{cccc}
\hline Variável & Coeficiente & Erro Padrão & Razão- t \\
\hline CONSTANTE & 3,4726 & 0,00790 & $439,5^{* * *}$ \\
GEN ${ }^{*}$ EDUC & 0,0364 & 0,00287 & $12,66^{* * *}$ \\
ETN*EDUC & 0,0767 & 0,00238 & $32,20^{* * *}$ \\
\hline R2 & \multicolumn{3}{c}{0,175} \\
\hline Estatística F & 1332,61 \\
\hline
\end{tabular}

** indicam significância a $1 \%(2,58)$.

Fonte: Elaborado pelos autores em 2017 a partir dos dados da PNAD 2015.

$7 \quad$ modelo 6 foi feito de maneira separada dos regressores dos modelos de 1 a 5 para que o resultado ficasse estatisticamente significativo a, pelo menos, $10 \%$. Quando foi feito o teste juntamente ao modelo 5, a variável ETN*EDUC deixou de ser significativa. $O$ intuito é verificar a sensibilidade das variáveis ETN e GEN na educação, por isso, foi mantido um modelo separado. 
Os resultados indicam a forte influência do gênero afetando a renda do indivíduo ${ }^{8}$. De acordo com o modelo $5^{9}$, homens ganham, em média e ceteris paribus, aproximadamente $18,41 \%$ a mais que as mulheres. Barros et. al (2001) também observaram que os homens são melhores remunerados por hora e, além desse fator, eles, em média, cumpre Am uma jornada de trabalho mais elevada que as mulheres, tornado a diferença de renda ainda maior.

O modelo 6 (Tabela 5) foi criado para analisar a diferença salarial entre homens e mulheres com o mesmo nivel de educação. Podemos verificar que em média, homens ganham $3,7 \%$ a mais que as mulheres com o mesmo nivel educacional. Fazendo um comparativo com os modelos de 1 a 5 , observa-se uma pequena variação de percentual na variável gênero com a inclusão de controles adicionais, tendo um pico de $22,5 \%$ no modelo 3 com a inclusão da variável CDM sobre condição de moradia do indivíduo. $O$ menor valor foi de $18,41 \%$ do modelo 5 já analisado.

A segunda variável de interesse a ser analisada é a etnia das pessoas (ETN). Uma especificidade da amostra do Estado do Rio Grande do Sul é que a grande maioria dos indivíduos entrevistados e que possuem renda são pessoas brancas, de acordo com a Tabela 6. O total de homens da amostra analisada corresponde à $55,53 \%$. Deste total, aproximadamente, $79,43 \%$ são brancos e $20,57 \%$ são negros. As mulheres representam $44,47 \%$ do espaço amostral, sendo, deste total, $81,66 \%$ mulheres brancas, contra apenas $18,34 \%$ de mulheres negras no Estado. Historicamente, o Estado do Rio Grande do Sul tem uma forte influência em migração e descendência europeia, o que pode talvez justificar o baixo número de negros dentro da amostra analisada.

Tabela 6 - Número de observações em cada grupo de interesse

\begin{tabular}{cccc}
\hline & BRANCOS & NÃO BRANCOS & TOTAL \\
\hline HOMENS & 5512 & 1428 & 6940 \\
MULHERES & 4538 & 1019 & 5557 \\
\hline TOTAL & 10050 & 2447 & 12497 \\
\hline
\end{tabular}

Fonte: Elaborado pelos autores em 2017 a partir dos dados da PNAD 2015.

Com base nos resultados do modelo 5 , brancos, em média e ceteris paribus, ganham aproximadamente $9,58 \%$ a mais por hora de trabalho. Os resultados do modelo 6, que analisa pessoas brancas e não brancas com o mesmo nivel de escolaridade, evidencia que, pessoas brancas recebem, em média e ceteris paribus, $7,97 \%$ a mais do que não brancos. Fazendo um comparativo entre os modelos de 1 a 5 , é notável que a variável ETN teve um aumento do percentual

8 Serão apresentados os percentuais reais pela equação: [exp(nominal) - 1]100.

9 Faz sentido a referência da análise sempre estar baseada no modelo 5 por ser o modelo mais completo do trabalho. Diferenças significativas e pontuais serão consideradas, mas o modelo 5 é tido como o "modelo final". 
do modelo 1 até o modelo 3, após uma leve redução nos modelos 4 e 5 . O maior percentual de discrepância do salário/hora em relação à etnia do indivíduo pode ser evidenciado no modelo 4, com um percentual de 10,15\% e o menor valor é visto no modelo 1 , com $7,94 \%$.

Todas as variáveis de controle apresentaram-se estatisticamente significantes, individualmente e conjuntamente, ao nível de $1 \%{ }^{10}$. A variável EDUC corresponde aos anos de educação do indivíduo, tendo um efeito, em média e ceteris paribus, de aproximadamente $8,68 \%$ de um maior salário/hora por ano de estudo das pessoas, o que condiz com a teoria do capital humano em maiores rendimentos para pessoas com maiores qualificações. Dos modelos de 1 a 5 , o maior percentual do impacto de um ano a mais de educação no salário/hora está no modelo 1, de $10,33 \%$ e o menor, no modelo 5 , que já foi citado na análise, de aproximadamente $8,68 \%$ de aumento salarial por hora por ano de estudo adicional.

A variável PERM é a relação do impacto da permanência do indivíduo no emprego atual e como isso se reflete no seu salário/hora, ou seja, tendo impacto positivo na renda individual devido ao conhecimento do trabalho, equipe, destreza, etc. $O$ modelo 5 evidencia que o tempo de permanência do individuo no trabalho atual causa um aumento em média, ceteris paribus, de 1,32\% no salário/hora. $O$ maior percentual foi encontrado no modelo 3 , de 1,42\% e o menor no modelo 2 , de aproximadamente, $1,23 \%$. É notório que a diferença percentual entre os modelos de 1 a 5 dessa variável é extremamente baixa.

As variáveis EXPER e EXPER ${ }^{2}$ são semelhantes a variável PERM, porém, a diferença é que ela não relatam apenas o tempo de permanência no trabalho atual, e sim toda a experiência profissional do indivíduo. É comum existirem casos de pessoas que possuam baixo tempo de permanência no emprego atual e possuem um vasto período de experiência profissional. A experiência profissional também é tida como benéfica fazendo sua relação com a renda individual, devido à experiências de vida e de trabalho, o trabalhador consegue agregar maior produtividade devido sua maior experiência. $\bigcirc$ modelo 5 aponta que cada ano de experiência a mais colabora com um salário/hora maior, em média e ceteris paribus, de $2,2 \%$, o que não difere muito também do percentual maior da análise do modelo 1 , de $2,7 \%$. O termo quadrático foi colocado na regressão para apontar qual o auge da experiência profissional em rendimentos marginais superiores de salário/hora, em outras palavras, até em que ponto a experiência profissional irá proporcionar ganhos marginais crescentes. Foi calculado que, com aproximadamente com trinta e três anos de experiência profissional, o indivíduo começa a obter ganhos marginais decrescentes de salário/hora, ou seja, o produto marginal da experiência profissional começa a ser decrescente em relação ao salário, o aumento salarial real tende a ser cada vez menor a partir de então.

10 Pode-se perceber que nos modelos 1 a 4, a significância da variável FILH (número de filhos) era de 5\%, portanto, com a inclusão da variável POT no modelo 5 , ela se torna estatisticamente significativa ao nível de $1 \%$. 
Para a variável FILH, os resultados da estimação do modelo 5 indicam que, em média, a pessoa recebe um salário hora menor em $2,64 \%$ a cada filho. Como - estudo não estimou equações separadas para homens e mulheres, esse é o resultado médio de ter um filho sobre o salário/hora de trabalho do individuo. Porém, conforme Wajnman (2016), ter filhos, tanto em idade pré-escolar quanto em idade escolar, resulta em efeitos diferentes para homens e mulheres. Para homens, ter cônjuge e ter filhos gera um aumento na sua participação econômica ativa (PEA). Para as mulheres o efeito é contrário, ter filho e/ou cônjuge, reduzem as chances de participação economicamente ativa.

Para a variável SET, os resultados indicaram que trabalhar no setor público aumenta o salário/hora, aproximadamente, $41,05 \%$ visto no modelo 5 , contra $36,8 \%$ do modelo 4 , sendo o menor percentual analisado. $\bigcirc$ estudo realizado por Becker e Kassouf (2011) para professores da rede pública e privada do ensino fundamental, evidenciou que a remuneração dos professores da rede pública de ensino é, em média, maior que na rede privada, principalmente quando se considera a remuneração recebida ao longo da vida.

A condição de moradia, CDM, apresenta uma relação positiva com o salário/ hora que, em média, é maior de $38,4 \%$ a $40,77 \%$ se o indivíduo mora em área urbana. A variável CUD diz respeito à condição na unidade domiciliar, significando que, em média, o indivíduo que é chefe de família, ganha um salário maior de $11,58 \%$ a $11,85 \%$, para o que não é chefe de família. $O$ indivíduo na posição de empregador (POT) ganha, em média, 52,8\% a mais de salário/hora que a pessoa não empregadora.

\section{Conclusão}

Esse estudo analisou as diferenças salariais de gênero e etnia no Estado do Rio Grande do Sul, com base nas informações da PNAD (2015). Constatou-se a existência de diferenciações salariais relacionadas à produtividade e também à elementos de cunho discriminatório, como gênero e etnia. Com base nos modelos apresentados, constatou-se que o salário médio dos homens ultrapassa em $18,41 \%$ os salários das mulheres e, em mesmo sentido, observa-se também uma diferenciação salarial entre autodeclarados brancos em relação aos negros, pardos e indígenas, em média, de 9,58\%. A vantagem salarial desses grupos também é observada quando são considerados indivíduos com o mesmo nível educacional.

De modo complementar, observou-se que o pior cenário discriminatório em relação ao gênero e etnia seria justamente para o caso das mulheres autodeclaradas não brancas. Afinal, contam com duplo vetor discriminatório, por serem de gênero feminino e não serem brancas. As variáveis que identificam a existência e quantidade de filhos, o tempo de experiência profissional e o nivel 
educacional também são determinantes importantes do salário do indivíduo.

Fica evidente, portanto, a necessidade do estabelecimento de políticas públicas no Estado do Rio Grande do Sul voltadas a igualdade étnica e de gênero nos rendimentos relacionados ao mercado de trabalho em funções semelhantes. Conforme visto na contextualização acerca do tema, parece haver uma tímida mudança neste cenário, porém ainda muito incipiente e, mais uma vez, salientando a necessidade de mecanismos efetivos no combate a essas desigualdades.

\section{Referências}

ALVES, T. VIEIRA, F. Uma Investigação Econométrica com Dados Cross Section dos Determinantes do Impacto da Crise Financeira Internacional (2008 - 2009) sobre o PIB. Universidade Federal de Uberlândia, 2011.

BARROS, R.; CORSEUIL, C. et al. Inserção no mercado de trabalho: Diferenciais por sexo e consequências sobre o bem-estar. Texto para discussão $n^{0}$ 796. Rio de janeiro, julho de 2001.

BECKER, K.; KASSOUF, A. Diferença salarial e aposentadoria dos professores do ensino fundamental. Economia aplicada, v. 16, n.1, 2011, pp. 77 - 104.

CAMPANTE, Filipe R.; CRESPO, Anna R. V. and LEITE, Phillippe G. P. G..Desigualdade salarial entre raças no mercado de trabalho urbano brasileiro: aspectos regionais. Rev. Bras. Econ. [online]. 2004, vol.58, n.2, pp.185-210.

CAVALIERI, C.; FERNANDES, R. Diferenciais de salários por gênero e cor: uma comparação entre as regiōes metropolitanas brasileiras. Revista de Economia Política, vol. 18, n¹ (69), janeiro - março/1998.

GIUBERTI, A. FILHO, N. Discriminação de rendimentos por gênero: Uma comparação entre o Brasil e os Estados Unidos. Março de 2005.

HENRIQUES, R. Desigualdade racial no Brasil: Evolução das condições de vida na década de 90. Texto para Discussão do Ipea, no. 807, 2001.

HOFFMANN, R.; LEONE, E. Participação da mulher no mercado de trabalho e desigualdade da renda domiciliar per capita no Brasil: 1981 - 2002. Nova Economia - Belo Horizonte, v.14, n.2, maio - agosto/2004.

IBGE. Instituto Brasileiro de Geografia e Estatística. Pesquisa Nacional por 
Amostra de Domicílios (PNAD), Rio de Janeiro, 2006. Disponível em: www. ibge.gov.br. Acesso em 10 de maio de 2017.

IPEA. Instituto de Pesquisa Econômica Aplicada. Boletim 23. Políticas Sociais: Acompanhamento e Análise. Assistência Social. Brasilia: IPEA, 2015.

LAVINAS, L. Empregabilidade no Brasil: Inflexões de gênero e diferenciais femininos. Texto para discussão $n^{0}$ 826. Instituto de Pesquisa Econômica Aplicada - IPEA. Rio de janeiro, setembro de 2001.

LEONE, E. O trabalho da mulher em regiões metropolitanas brasileiras. In: PRONI, M. W.; HENRIQUE, W. (Org.). Trabalho, mercado e sociedade. O Brasil nos anos 90. São Paulo: Editora UNESP; Campinas: Instituto de Economia da UNICAMP, 2003.

LOUREIRO, P. Uma resenha teórica e empírica sobre economia da discriminação. RBE, Rio de janeiro, janeiro - março/2003 pp. 125 - 157.

SEN, A. Desenvolvimento como Liberdade. São Paulo: Companhia das Letras, 2010.

SILVA, N.; KASSOUF, A. Mercado de trabalho formal e informal: Uma análise da discriminação e da segmentação. Nova Economia - Belo Horizonte, v. 10, n.1, julho de 2000.

SOARES, S. O perfil da discriminação no mercado de trabalho - Homens negros, mulheres brancas e mulheres negras. Texto para discussão $n^{0} 769$. Instituto de Pesquisa Econômica Aplicada - IPEA. Brasilia, novembro de 2000.

WAJNMAN, S. "Quantidade" e "Qualidade" da participação das mulheres na força de trabalho brasileira. Associação Brasileira de Estudos Populacionais, p. $45-58,2016$.

WOOLDRIDGE, J. M. Introdução à Econometria. São Paulo. Cengage Leraning. $4^{\circ}$ edição, 2010.

Recebido em junho de 2018

Aprovado em setembro de 2018 